\title{
Le degré in-fini de l'écriture : la prose d'Éric Chevillard
}

\author{
Thangam Ravindranathan \\ Brown University
}

De curieux êtres peuplent l'œuvre d'Éric Chevillard. Tels des points de déliaison dans la matière du monde, ce sont des entités insaisissables, frôlant l'impensable aussi bien selon les lois du «réel» qu'à l'égard de celles qui, habituellement, assurent à une diégèse son horizon de référence, de cohérence internes. Ainsi de Palafox, protagoniste éponyme d'un roman de 1990, créature en métamorphose continuelle, que des zoologues parviennent un temps à mettre en cage sans pour autant pouvoir l'identifier : 
Les quatre hommes font cercle autour de la cage de verre où l'animal exposé se tient enfin tranquille. Zeiger examine ses yeux, ses naseaux, son aigrette, Camberlin son flanc droit, Baruglio sa croupe, Pierpont sa main gauche, on tourne, Pierpont examine ses yeux, son groin, sa barbiche, Zeiger son bras droit, Camberlin son dard, Baruglio son aile gauche, on tourne, Baruglio examine ses yeux, son bec, ses antennes, Pierpont sa nageoire droite, Zeiger sa queue aplatie en truelle, Cambrelin son flanc gauche, on tourne, Cambrelin examine ses yeux, ses barbillons, ses cornes, Baruglio son ouïe droite, Pierpont ses rectrices, Zeiger son bras gauche, on se regarde, certes, il faudrait pour bien faire disséquer Palafox. (Chevillard, 1990, p. 21-22)

Le manège complexe, dans ses effets habilement cubistes, joue du temps et de l'espace de la rotation des zoologues pour accumuler l'hétérogène. Les parties du corps de Palafox tiennent diversement du poisson, de l'oiseau, de l'insecte, du cheval, du porc et de l'humain; le tout ne pouvant faire identité, ni dans le temps, ni dans l'espace, ni dans le nombre. Sous quelles conditions un tel vertige fictif vient-il occuper, préoccuper, parasiter les structures d'un récit et, partant, l'ordre du « réel »? Que peut bien signifier un tel être quant au passage entre réel et fiction, entre mots et choses?

Prenons encore la cicatrice qui défigure le visage de Crab, entité nébuleuse s'il en est, personnage pour ainsi dire principal de deux romans de Chevillard, La Nébuleuse du crabe et Un fantôme. On nous donne au sujet de cette cicatrice une série d'explications :

cette balafre hideuse lui fut infligée au cours d'une rixe, par son adversaire armé d'un biface de silex tranchant, au cours d'un combat, par l'envahisseur armé de son glaive, sur le champ de bataille, par l'ennemi armé de son sabre, de sa lance, de son tomahawk, de sa baïonnette, cette balafre est la trace d'une gifle formidable qu'il reçut de sa mère, il y a longtemps, dans sa 
tendre enfance, il ne sait pas pourquoi, mais il ne l'avait pas volée. (Chevillard, 1995, p. 55)

\section{Surplus du signifiant}

Il s'agit de ce que Gilles Deleuze qualifiait, commentant par exemple certains écrits de Samuel Beckett, de «disjonctions inclusives », soit des propositions de sens divergentes qui, logiquement, devraient réciproquement s'exclure, mais qui peuvent être amenées par moments à coexister, faisant alors, écrivait Deleuze, «bégayer la langue» (1993, p. 138-139). On note en effet l'absence, pour ce qui est de la phrase-balafre de Crab, de toute conjonction pouvant expliciter le rapport (succession, alternance, cumulation?) ou réparer l'incompatible des propositions. Il en va alors, comme lors du tour des zoologues de Palafox, d'une spatio-temporalité narrative ellemême affectée d'une non-identité interne ou, en tout cas, en assumant la charge. La série de propositions, faisant tourner l'événement sur une pluralité irréductible, l'ouvre sur un fond indécidable. La cicatrice en question, objet d'une surdétermination causale, se fait le signe d'un corps débordé par le verbe, excédé par une narrativité qui lui impute trop d'origines, l'enrôle dans trop de récits. Si on en étend l'économie symbolique, elle vient dire un monde où il y a plus de récits d'événements que de corps pour les vivre, plus de mots que de choses.

L'homme dispose face au monde, écrivait Derrida, d'un surplus du signifiant. L'univers de Chevillard est travaillé de manière quasi systématique par un excédent du narratif sur le référentiel, phénomène que vient encore illustrer de manière exquise l'irrésolution du premier cri de Palafox : «Un matin au 
réveil, il fit entendre son cri, comment dire, une espèce de piaillement, ou plutôt de miaulement, ou plutôt d'aboiement, ou plutôt de mugissement, nous y sommes presque, de rugissement, ou plus exactement de barrissement, oui, c'est le mot, une espèce de piaillement. » (Chevillard, 1990, p. 15) S'ouvrant en un spectre de cris d'autant d'espèces - l'oiseau, le chat, le chien, le ruminant, le lion, l'éléphant —,la phrase (phrase-cri) fait mine de s'approcher progressivement (« plutôt»; « nous y sommes presque », « ou plus exactement ») du terme exact pour revenir au final à l'oiseau du début (à moins qu'il ne s'agisse d'un autre oiseau!), comme pour témoigner d'un axe paradigmatique et, dès lors, d'une topologie de l'être disposée plutôt en boucle. Ces moments, chez Chevillard, fascinent par leur insertion, dans la prose (mot qui en latin signifie droit, direct), dans la syntaxe du récit, de points de circularité, d'effets-tourniquet où l'apparente résolution (« oui, c'est le mot») cache mal ce qui est plutôt l'indomptable loi du cercle: parfaite et infinie indécidabilité du sens, irrecouvrable origine (où commence un cercle et où se terminet-il?). "Le bégaiement créateur», écrivait Deleuze, " est ce qui fait pousser la langue par le milieu, comme de l'herbe, ce qui fait de la langue un rhizome au lieu d'un arbre, ce qui met la langue en perpétuel déséquilibre: Mal vu mal dit (contenu et expression). » (1993, p. 140, je souligne). Des unités de sens qui, dans la langue en équilibre, dans le réel qu'elle ordonne, seraient irréconciliables - car violant une taxinomie qui, pour être syntaxique, n'est pas moins ontologique - , sont amenées, en ces lieux que marque Palafox, à cohabiter, telles des portes battantes, à la faveur d'une sorte de ou inclusif. Économie singulière, où les « bégaiements » viennent signaler autant de points de décrochage entre signe et ontos d'une transaction 
impure entre le monde et les représentations. Or, c'est précisément dans ces régions où les mots et les choses ne s'équivalent plus, où leurs frontières ne coïncident plus, dans cet excédant, donc, ces plis qu'instaure la relation symbolique (lieux nécessairement désavoués du langage nominaliste, réaliste), que prennent chair et puissance les curieuses créatures de Chevillard.

La langue est conduite là vers sa limite, son asyntaxie. Non que la syntaxe en tant que telle soit malmenée ; c'est plutôt l'ordre des mots et des choses, les coordonnées de l'axe référentiel qui sont redistribués par ces réfractions en « éventail » du discours. Les disjonctions inclusives écartèlent l'être; sans nom propre pour les tenir ensemble, Palafox ou Crab auraient explosé, croirait-on, d'être tant de choses. Mais on est devant une écriture qui, profitant des dérives des mots eux-mêmes, exploitant leur part de dehors, détourne les définitions pour détromper les identités, pour les avérer différences. Diogène des lettres contemporaines, Chevillard nous propose pour des hommes autant de poules déplumées, jouant de ces lieux de fuite des catégories, de ces pentes par où ladite propriété au contraire ex-proprie, éloigne du propre. Ainsi de ce passage d'Un fantôme concernant le lapin :

Le lapin est certes facile à dépouiller, on ne peut pas se plaindre, il le serait encore plus s'il avait au lieu de cette fourrure qui l'habille une peau de banane. Il est certes facile à découper, soyons juste, il le serait plus encore s'il était constitué de quartiers comme l'orange. Sa chair est tendre, indéniablement, elle le serait davantage si elle était tout en poulpe comme la fraise. Tout de même, la vie de Crab serait bien simplifiée si le lapin était un fruit. (p. 94-95) 
L'évaluation du lapin selon ses propriétés utiles à l'homme le situe à un tel désavantage que, pour finir, force est de conclure qu'il aurait plus ou mieux été lapin en étant fruit. La somme de telles propriétés rapporte le lapin non pas à lui-même mais à d'autres. Combien de propriétés du lapin faudrait-il nommer pour le distinguer de toute autre chose et nommer enfin sa singularité? Comment sortir de l'impropre, des parasitages, de ces glissements sur l'axe paradigmatique, de la porosité des signes? Fallait-il reconstituer le monde entier pour dire proprement le lapin? Comment rejoindre l'être? Le lapin est pris, certes, dans les mailles d'autres logiques régnantes, celles de l'utilité, de l'échange, celle d'un système de substitutions où la substance est perdue de vue. Critique de la société de consommation, peut-être, Chevillard est avant tout un maître des ressources de la fiction et de ce qui, en elle, permet de démonter le réel. « [L]e monde existe d'abord pour nous dans la fiction de la langue», dit-il. «En le nommant ou en le renommant, j'agis au moins sur cette illusion. » (2007, p. 105) Reprochant au roman de trop «se conform[er] au principe de réalité », l'écrivain voit en lui plutôt « l'occasion de formuler des hypothèses divergentes, de faire des expériences, d'éprouver de nouvelles façons d'être» (p. 96). Dans son lapin-fruit, une ancienne irresponsabilité et le propre de la poîesis, face au réel, s'assument et jouissent : sans pression du modèle, justiciable d'aucune des contraintes du monde, l'être de la fiction peut accueillir toutes les propriétés. Il se laisse emporter le long d'une ligne de fuite, celle de la folie du signe. 


\section{Le tiers inclus}

Il se trouve que Crab souffre d'une affliction insolite : ayant une langue de cire, s'il se tait, "sa langue aussitôt se figera définitivement dans sa bouche. Il parle donc, il dit n'importe quoi, une chose et son contraire, que l'éléphant devrait se vêtir de daim, et on s'imagine qu'il délire, tandis qu'il lutte contre la mort. » (Chevillard, 1993, p. 15) Cette affliction, qui ne dure que l'espace d'un fragment et que ne confirme nulle suite dans le récit, en fournit cependant une clé stylistique, thématique, voire logique. Car, dans une parole qui ne peut s'interrompre et dont l'urgence est moins dans son propos que dans son débit continuité interdite de rupture -, le langage s'affranchit du poids du référent pour se faire pure signifiance, référentialité fantomatique. Or, voilà qui nomme la nature même de cette diégèse qui ne cesse de se recouper, de se contredire, en proie à un délire du signifiant. En effet, dire sans cesse, c'est dire sans les silences que composerait, qu'imposerait la logique même de la parole. Silences non pas à vrai dire temporels mais symboliques, c'est-à-dire ceux de toutes ces zones d'exclusion que produit chaque proposition, de tout ce qui, pour qu'une parole soit vraie, doit être congédié à l'ombre de l'étant, raturé, marqué comme étant faux ou impertinent. En ce sens, il s'agit, chez Chevillard, d'une parole qui ne sait pas tout à fait ce qu'elle dit ou, en tout cas, ne le sait pas assez pour exclure ce qu'elle ne dit pas, menant donc à une absence d'horizon d'organisation verbale et ontologique du référent donné. De quoi expliquer sans doute la facture fragmentaire de ces récits, enchaînant des propositions qui ne tolèrent pas d'être lues ensemble, mais qui, néanmoins, se laissent suivre avec, pour alibis référentiels ou effets de langage schizophrènes, un monde toujours recommencé. Ainsi, Palafox peut enchaîner les aspects d'oiseau 
mouche, d'éléphant et de cervidé ; Crab est caractérisé aussi bien par «sa laideur effrayante » que par «la beauté régulière de ses traits » (1995, p.11); une taxinomie fantaisiste sur le plan des mots entraîne une ontologie inédite, irréconciliable, sur le plan du monde-référent. « Je me débats surtout contre ce qui est pourtant le propre du langage : produire des énoncés où le sens se fige », dit encore Chevillard. «Je viole sans cesse le principe de non-contradiction qui relève ordinairement de l'engagement tacite de l'écrivain envers son lecteur. » (2007, p. 102)

«Le logos (ou le temps de la logique) », écrit Derrida, est « dominé par le principe de la non-contradiction, fondement de toute métaphysique de la présence» (1967, p. 321). Ce qu'il rappelle par là est une tradition remontant à Platon, qui, on le sait, bannissait la poésie de la cité parce qu'elle nommait le penser de l'impensable, à savoir ce qui « ruine la discursivité » (cité par Badiou, 1998, p.33). Julia Kristeva, pour sa part, commente ainsi ce verdict platonicien, qui assure la séparation du réel de son autre intime et obscur et, par là même, fonde la rationalité du signe :

Chez Platon (Le Sophiste), le propre du discours (Logos) étant d'identifier, d'être une présence à soi, il ne peut inclure le terme nié, c'est-à-dire le terme non-identique, le terme absent, le terme non-existant, que comme une éventualité (comme une non-existence) à partir de laquelle nous pouvons dire ce qui est l'autre du nié : le même. En d'autres termes, la logique de la parole implique que la parole soit vraie ou fausse («ou» exclusif), même ou autre, existante ou inexistante, mais jamais les deux à la fois. Ce qui est nié par le sujet parlant, ce qui est réfuté par lui, constitue l'« origine » de sa parole (puisque le nié est l'origine de la différenciation, donc de l'acte de la signification), mais ne peut participer à la parole que comme exclu d'elle, essentiellement autre par rapport à elle et par 
conséquent marqué par un indice de non-existence qui serait l'indice de l'exclusion, de la fausseté, de la mort, de la fiction, de la folie. (1969, p. 188)

L'exclusion, la rature originelle, d'un indicible, d'une parole folle ou inadmissible fonderait ainsi (et à chaque prise de parole) la possibilité de dire. Si le réel et les récits qui le consignent reposent sur le principe de la non-contradiction, c'est précisément en ce que la contradiction première que doit écarter, nier l'ordre du réel - et qui constituerait dès lors, pour Kristeva, «l'origine » de ce réel — est sans doute celle qui nomme une part d'indifférenciation entre existence et inexistence. Dans le logos, dans le réel qu'il assure, quelque chose est ou n'est pas, «mais jamais les deux à la fois ». Il n'y a pas de tierce possibilité : c'est ce qu'on appelle le principe du tiers exclu. Certes, la fiction est ce tiers, exclu de la cité parce que ménageant la possibilité que quelque chose soit et ne soit pas en même temps. C'est ce que disait Paul Ricœur de la métaphore vive au fondement de toute poîesis: Aixo era y no era. Or, cette puissance, la fiction peut l'exploiter de manière littérale et dans son détail (même si, dans les faits, seule le fait une littérature mineure). Car qu'est-ce qui l'empêche de dire une chose et puis son contraire? C'est cette insistance du tiers qu'incarnent comme par principe Palafox ou Crab, tels des morceaux de fiction - de fictionnalité - pure, donnant leurs noms à des propriétés irréconciliables. En eux jusqu'à la mort et la vie s'entrecoupent et se confondent: on apprend, parmi d'autres énoncés irréconciliables, que Crab a été écrasé par un autobus (Chevillard, 1995, p. 48), qu'il est mort à la naissance (p. 36) ou « dans son œuf » (p. 52), ou encore qu'il a été déclaré mort et incinéré, alors qu'il était et continuait d'être vivant jusque dans sa forme empoudrée (p.121-122). Pas de quoi 
mettre en péril, cependant, le récit des exploits et bizarreries du personnage, comme si la mort ne constituait, au même titre que tout autre développement narratif, que l'une parmi plusieurs propositions pouvant lui permettre d'avancer dans le récit. Vers la fin d'Un fantôme, les médecins tiennent encore Crab pour mort: «Ses interventions sont trop rares pour ébranler leur conviction, son pouls est trop capricieux. Cet homme est mort, répètent-ils, son décès remonte au moins à trois ou quatre ans. N'exagérons rien. Crab n'est pas en vie, indéniablement, mais de là à prétendre qu'il est mort? Crab ne sait plus lui-même. Peutêtre, après tout? » (p. 107)

Si Crab, tout comme Palafox, est dispensé de coïncider avec lui-même, c'est que, à chaque moment, ces personnages ne font qu'occuper une place prédicative, soit «profit[er] en parasite de structures existantes » (Chevillard, 1990, p. 25). Ces personnages, leur créateur les assimile à «des pronoms personnels nouveaux qui viennent parasiter la langue et [qui] profitent en effet de ses extraordinaires ressources, de son efficacité terrible, pour se développer selon leur loi propre » (Chevillard, 2007, p. 98). Moment peut-être de se poser une question qui, elle-même métaphorique, ne saurait susciter comme réponse qu'une métaphore : quelles structures, au juste, pré-existent à la fiction? À quoi ressemble le « réel » que trouve et parasite l'être fictif? Sous quels traits le réel apparaît-il à celui-ci? Autrement dit, qu'est-ce que le réel, vu du point de vue de la fiction? Sans le savoir, toute fiction propose peut-être à de telles questions une réponse, un récit de cette rencontre, de ce greffage par où, s'enlevant au réel, aura pris consistance un être de fiction. Celles de Chevillard portent les traces d'un monde trouvé intact, tout fait, mais curieusement gelé, dépeuplé. Dans ce manège abandonné, des êtres n'ayant aucune histoire et 
aucune forme propre jouent alors à occuper les places, voire toutes les places. Serait-ce parce qu'ils occupent seuls le monde, sont seuls dans leur ontologie fictive, hétérogène au réel, venant peupler celui-ci et, pourtant, ne le touchant pas, tels des fantômes? Ainsi, Crab est l'inventeur de toutes choses (Chevillard, 1995, p.15-17), adepte de tous les sports et de toutes les disciplines (p. 20-21) ou encore la cible, la victime de tout événement :

c'est toujours lui que le froid engourdit, que le feu brûle, et s'il est en ce monde quelqu'un à qui le brouillard dissimule toutes choses, qui va souffrir de la soif jusque dans le désert, ce quelqu'un, vous ne risquez rien à parier que c'est Crab, c'est le visage de Crab qui se ride quand le temps passe, ce sont ses facultés qui s'émoussent, et l'homme qui va mourir un jour, vous verrez que ce sera lui, encore Crab, desservi par le sort jusqu'au bout, victime une dernière fois de sa malchance. (p. 11)

Palafox, non content d'assumer toutes les formes zoologiques, est selon certains responsable de toutes les violences commises contre l'être humain depuis des millénaires (voir p. 88-90). L'héritage de Michaux est indéniable — songeons notamment à «Encore des changements » (Mes propriétés) et « Naissance » (Un certain Plume) : le monde se déplie comme un gigantesque catalogue de possibles ontologiques, de vides faits d'autant de prédicats plus ou moins humains en quête de sujets.

\section{Préhistoire ou le réel ex-proprié}

La métaphore du manège abandonné n'a pas pour fonction d'exclure d'autres personnages des récits qu'occupent Palafox et Crab, elle rend seulement compte de ce que ceux-ci s'y inscrivent comme seuls corps mobiles, énergiques, capables et 
susceptibles de tout, contre un fond à tendance autrement fixe, voire cadavérique. Corps vides, corps excessifs, «[i]ls occupent un point de vue. Ils adoptent une posture», dit l'auteur de ses personnages. "Ils servent mon propos. Ils conduisent la charge. » (Chevillard, 2007, p. 103) Parce que Palafox et Crab n'ont pas de lieu propre, parce que, mal vus, mal dits, ils esquissent des ontologies scandaleuses, une force normative sociale (que décrit et double avec ironie le récit) les traque, s'acharne à les rendre responsables d'un acte, à décider de leur place, à les confiner en des rôles, bref, à les constituer en sujets. Tous deux sont soupçonnés de crimes, coïncidence qui ne fait que confirmer la raison et l'angoisse économiques au cœur de la pensée du sujet : pendant que Crab est retenu en prison durant quelques jours, le taux de meurtres et de méfaits dans le monde, habituellement assez haut, diminue (1993, p. 45). Les autorités en concluent qu'il était responsable d'un grand nombre de ces crimes et décident dès lors de le garder en prison. Le raisonnement à l'endroit de Palafox n'est pas différent: puisqu'on n'a pas trouvé les responsables de beaucoup de violences commises depuis la nuit des temps contre l'homme et puisqu'il est impossible d'établir un alibi temporel ou spatial pour Palafox permettant de l'en innocenter (on n'arrive pas à savoir quand et où il est né, et quel a pu être son périmètre géographique), on ne peut exclure l'hypothèse que c'est bien à lui seul que correspondent tous ces crimes restés anonymes. Purement distributionnelle, allocative, «némétique » (némein : partage d'espace, d'où nous viennent aussi bien le terme némésis que celui d'économie), c'est cette logique même qui gère la pensée législative du sujet, logique par excellence narrative, herméneutique du récit policier (qui commit ce crime?), mais qu'on voit organiser de manière tout 
aussi intime le vigile d'un réel contemporain pathologique (terrorisme, épidémies). La pensée du némein, du partage, de la loi, est ce qui distribue et constitue les lots des sujets.

Or, persister avec cette illusion grammaticale du sujet (comme aurait dit Nietzsche), c'est, comme l'attestent au contraire ces solutions économiques absurdes (Palafox, Crab absolument coupables), encourir un péril inverse, celui de buter contre un ordre d'événements préhistoriques, c'est-à-dire sans sujet nommable ou, en tout cas, sans sujet singulier. «[L]'Histoire commence quand les jeux sont faits», estime Chevillard. «La préhistoire est au contraire l'âge où tout était possible. L'écrivain qui saurait s'y transporter jouirait soudain d'une liberté absolue pour rêver autre chose que ce qui fut, que ce qui est. Puisque rien n'était écrit, tout reste à écrire, partant de l'origine, tout, sauf ce qui est advenu... » (2007, p. 96-97) La préhistoire ne nomme-t-elle pas au fond un temps de propriété trouble (si l'on entend propriété comme relation numérique, unité d'une économie, d'une grammaire, qui distribue les prédicats entre les sujets et ainsi différencie les sujets, et le tout sans reste), où l'on ne saurait plus identifier, pour chaque propriété ou prédicat, un sujet unique, ou encore, pour un sujet supposément unique, un prédicat qui lui serait propre? Temps immémorial ("Mes romans voudraient plutôt instaurer un temps hors de l'Histoire» dit encore Chevillard (p. 96)), qui vient figurer à l'extrême le lieu d'une indistinction entre sujet et objet. Dans un bel essai, Bruno Blanckeman dégage «toute l'aberration logique et sémantique» que vient opérer la préhistoire dans un roman de Chevillard intitulé précisément Préhistoire (1994, encadré donc, comme le note Blanckeman, par les deux récits de Crab). Car la préhistoire ne peut exister sauf à invalider le sens même de l'histoire; l'histoire ne peut 
qu'en se démentant exclure de son champ une période au titre qu'elle lui serait antérieure. Plus encore, la préhistoire, comme " la part effacée de la mémoire », "ne fonctionne plus comme ce point-zéro à partir duquel il devient possible d'historiciser un devenir humain, mais comme un point-gouffre qui aspire toute construction rétrospective et rend impossible toute pensée historique» (2004, p. 168, 170). La préhistoire vient inscrire une amnésie et une indifférenciation à la place de l'origine. C'est le «réel » anthropologique qui est ainsi exposé pour être, à l'instar de toutes les fictions, fondé à partir d'une "énigme originelle», d'un «lieu de vide» (p. 168). Préhistoire donne pour finir la parole à l'homme préhistorique lui-même, parole par excellence paradoxale qui vient dire à la première personne et au futur le difficile avènement du sujet humain :

C'est un projet ambitieux, peut-être chimérique, mais dont je ressens confusément la nécessité pour moi comme pour mes semblables. Cette activité nouvelle nous permettra d'exprimer notre différence, enfin nous oserons revendiquer notre spécificité au sein de toutes les espèces vivantes, nous lutterons efficacement contre cette condition inférieure, humiliante, à laquelle nous condamnent notre défaut d'instinct et notre faiblesse constitutionnelle. Nous aurons nos caractéristiques exclusives, nous aussi, notre originalité, nos rites, nos parades, nos pavanes, nos repères dans le temps et dans l'espace, nous cesserons de nous définir par nos manques et nos infirmités. Nous sortirons du flou. Nous prendrons corps. L'être humain sera reconnu, admis, identifiable, irréductible... (Chevillard, 1994, p. 169-170)

Parole à la fois prophétique et ironique (se proférant d'un lieu hors de l'inscription), c'est elle qui, comme un acte performatif sans assise, sans lieu, seule dit l'humain et marque le seuil de son avènement. Elle nous rappelle que nos récits, notre réel, s'écrivent et continuent à tout instant de s'écrire à partir de ce seuil paradoxal, de ce temps d'avant l'écriture. Alors, l'on peut 
revenir pour conclure à l'étrange non-identité à soi affectant les êtres de Chevillard et se demander si ces derniers ne viennent pas précisément, en désignant un temps hors de l'histoire et de la grammaire du sujet, renvoyer la supposée identité à soi du réel à sa dé-raison, à la parole niée qui la fonde. Le "tiers" infiltre ces récits, on l'a vu, comme corps n'ayant aucune forme fixe, aucune propriété sienne. Or, il faudrait prolonger le propos de Blanckeman pour considérer en quoi le préhistorique de Chevillard n'est pas seulement anthropologique, psychanalytique ou encore littéraire, mais encore ce qui vient « occuper», rouvrir au jeu, la discontinuité fondatrice opérée par l'institution d'une barre séparant le signifié du signifiant ou encore le biologique du social. Les créatures protéiformes de Chevillard viendraient alors figurer des corps préhistoriques au sens où ils seraient antérieurs à une histoire de la symbolisation, de la législation du sens. Dans la mesure où les distinctions syntaxiques et, partant, toute l'organisation de l'ordre symbolique procèdent d'une première législation des corps, d'une première taxinomie opérée sur les êtres, interdisant à l'oiseau / mouche d'être éléphant et à un je d'être un toi, ces corps viennent rappeler tout ce que le réel doit oublier pour être le réel, un monde de corps sans formes achevées et sans frontières, une sorte de réel à l'état infinitif, infini, d'un possible qui n'est pas réduit à ce qui est, mais continue à s'exercer selon toutes ses déclinaisons. "L'histoire n'est pas faite partout», écrit Chevillard. "Il reste des lieux dérobés où le temps préhistorique s'accumule.» (1994, quatrième de couverture) Telle l'enfance, ce temps qui, selon Lyotard, ne passe pas, , la préhistoire de Chevillard est comme ce qui au cœur du réel continuellement le disperse, le rappelle à ce qu'il nie et qui le fonde. 


\section{Bibliographie}

BADIOU, Alain. (1998), Petit Manuel d'inesthétique, Paris, Seuil.

BLANCKEMAN, Bruno. (2004), «De la préhistoire à l'aprèshistoire (Au sujet d'Éric Chevillard) », dans André BENHAÏM et Michel LANTELme (dir.), Écrivains de la préhistoire, Toulouse, Presses universitaires du Mirail, p. 159-176.

Chevillard, Éric. (2007) «Des crabes, des anges et des monstres », entretien avec Mathieu LARNAUDIE, dans François BÉGAUdEAU et. al. (dir.), Devenirs du roman, Paris, Naïve, coll. «Inculte », p. 95-109.

—. (2004) «Questions de préhistoire», entretien avec André BENHAÏM, dans André BenhaÏM et Michel LANTElme (dir.), Écrivains de la préhistoire, Toulouse, Presses universitaires du Mirail, p. 177-190.

—. (2003 [1990]), Palafox, Paris, Minuit,

—. (1993), La Nébuleuse du crabe, Paris, Minuit.

—. (1994), Préhistoire, Paris, Minuit.

—. (1995), Un fantôme, Paris, Minuit.

Deleuze, Gilles. (1993), Critique et clinique, Paris, Minuit.

DERRIDA, Jacques. (1967), L'Écriture et la différence, Paris, Seuil.

KRISTEVA, Julia. (1969), Seméiotikè. Recherches pour une sémanalyse, Paris, Seuil. 


\title{
Résumé
}

Deleuze relevait chez Beckett des «disjonctions inclusives", soit des sélections paradigmatiques logiquement incompatibles qu'une langue « bégayante » fait cependant cohabiter. La prose d'Éric Chevillard travaille cette limite de la langue. Ainsi, le protagoniste de Palafox (1990) est à la fois papillon, reptile et éléphant; Crab (Un fantôme, 1995) se distingue à la fois par «sa laideur effrayante » et « la beauté irrégulière de ses traits ». Une taxinomie fantaisiste sur le plan des mots entraîne une ontologie irréconciliable au niveau du monde-référent. Nous étudions ici les modalités par lesquelles ces corps irrationnels parasitent les structures du discours et, partant, celles du « réel ».

\begin{abstract}
Deleuze had noted Beckett's "inclusive disjunctions", that is, logically incompatible paradigmatic choices which could nonetheless coexist in a "stammering" language. Éric Chevillard's prose works this limit of language. Thus the protagonist of Palafox (1990) is at the same time butterfly, reptile and elephant, Crab (Un fantôme, 1995) is distinguished both by his "frightening ugliness" and the "irregular beauty of his features"; a fantastical taxonomy of words engages an irreconcilable ontology on the level of world-referent. I reflect here on the modalities whereby such irrational bodies parasitically occupy the structures of discourse and, thereby, of the "real".
\end{abstract}

